\title{
Evaluation of Serum Cystatin C as a Marker of Early Renal Impairment in Patients with Liver Cirrhosis
}

\author{
Mahmoud Omar, ${ }^{1}$ Wael Abdel-Razek, ${ }^{1}$ Gamal Abo-Raia, ${ }^{2}$ \\ Medhat Assem, ${ }^{1}$ and Gasser El-Azab ${ }^{1}$ \\ ${ }^{1}$ Department of Hepatology, National Liver Institute, Menoufia University, Shebeen El-Kom, Egypt \\ ${ }^{2}$ Department of Clinical Pathology, National Liver Institute, Menoufia University, Shebeen El-Kom, Egypt \\ Correspondence should be addressed to Gasser El-Azab; g.elazab@gmail.com
}

Received 13 July 2015; Revised 17 September 2015; Accepted 17 September 2015

Academic Editor: Daisuke Morioka

Copyright (C) 2015 Mahmoud Omar et al. This is an open access article distributed under the Creative Commons Attribution License, which permits unrestricted use, distribution, and reproduction in any medium, provided the original work is properly cited.

Background. Serum cystatin C (CysC) was proposed as an effective reflection of the glomerular filtration rate (GFR). However, its role in patients with liver cirrhosis has not been extensively verified especially in the detection of early RI. Patients and Methods. Seventy consecutive potential candidates for living donor liver transplantation with serum creatinine $(\mathrm{Cr})<1.5 \mathrm{mg} / \mathrm{dL}$ were included. CysC, Cr, and estimated GFR [creatinine clearance (CCr), Cockcroft-Gault formula (C-G), MDRD equations with 4 and 6 variables, CKD-EPI-Cr, CKD-EPI-CysC, and CKD-EPI-Cr-CysC] were all correlated to isotopic GFR. Early RI was defined as GFR of $60-89 \mathrm{~mL} / \mathrm{min} / 1.73 \mathrm{~m}^{2}$. Results. Patients were $25.7 \%$ and $74.3 \%$ Child-Pugh classes B and C, respectively. GFR was $\geq 90$, $60-89$, and $30-59 \mathrm{~mL} / \mathrm{min} / 1.73 \mathrm{~m}^{2}$ in $31.4 \%, 64.3 \%$, and $4.3 \%$ of the patients, respectively. All markers and equations, except C-G, were significantly correlated to GFR with CKD-EPI-Cr-CysC formula having the highest correlation $(r=0.474)$ and the largest area under the ROC curve (0.808) for discriminating early RI. At a cutoff value of $1.2 \mathrm{mg} / \mathrm{L}$, CysC was $89.6 \%$ sensitive and $63.6 \%$ specific in detecting early RI. Conclusion. In patients with liver cirrhosis, CysC and CysC-based equations showed the highest significant correlation to GFR and were measures that best discriminated early RI.

\section{Introduction}

Renal impairment is a common finding in patients with chronic liver disease; it has a huge impact on the patients' survival [1]. Moreover, the severity of renal dysfunction increases with the advancement of liver cirrhosis and portal hypertension [2]. Therefore, close follow-up of the renal function in patients with liver cirrhosis is mandatory and markers of early renal impairment are priceless in these patients [2].

Using the current markers and equations of the renal function in cirrhotic patients can be challenging. Serum creatinine, the most widely used marker, may underestimate renal impairment in patients with liver cirrhosis. Decreased hepatic production of creatine, reduced muscle mass, and malnutrition account for an increased gap between serum creatinine levels and the actual renal function [3]. High serum bilirubin levels may also interfere with the analytical methods of serum creatinine measurement, although this is no more a problem after using the modern Jaffe method autoanalyzers [4]. Inulin clearance, the standard method for measuring the glomerular filtration rate (GFR), is costly and impractical as it requires 24-hour urinary catheterization [5]. Isotopic renal scans are not less costly; they cannot be used for repeated measurements that are needed in such patients. Creatinine clearance tends to overestimate the GFR and requires accurate urine volume measurement [6]. Based on serum creatinine, Cockcroft-Gault formula and modification of diet in renal disease (MDRD) equations are of limited value in cirrhotic patients; they overestimate the GFR as well [7].

Serum cystatin $\mathrm{C}(\mathrm{Cys} \mathrm{C})$ has been proposed as a novel biomarker of the renal function [8]. Several studies have reported its value in different sets of patients [9-16]. However, only few studies have evaluated the role of serum CysC in patients with liver cirrhosis [17-19] and none of these studies have studied its role in detecting early renal impairment in 
TABLE 1: GFR-estimating equations used in the study.

\begin{tabular}{|c|c|}
\hline Cockcroft-Gault formula & 140 - age $($ years $) \times$ weight $(\mathrm{kg}) /(\mathrm{Scr} \times 72) \times 0.85$ if female \\
\hline $\begin{array}{l}\text { MDRD equations using } 4 \\
\text { variables }\end{array}$ & $186 \times \mathrm{Scr}^{-1.154} \times$ age $(\text { years })^{-0.203} \times 0.742$ if female $\times 1.212$ if black \\
\hline $\begin{array}{l}\text { MDRD equations using } 6 \\
\text { variables }\end{array}$ & $\begin{array}{l}170 \times \mathrm{Scr}^{-0.999} \times \text { age }(\text { years })^{-0.176} \times \mathrm{BUN}(\mathrm{mg} / \mathrm{dL})^{-0.170} \times \text { albumin }(\mathrm{g} / \mathrm{dL})^{0.318} \times 0.762 \text { if female } \times \\
1.180 \text { if black }\end{array}$ \\
\hline $\begin{array}{l}\text { The CKD-EPI creatinine } \\
\text { equation }\end{array}$ & $\begin{array}{l}141 \times \min (\mathrm{Scr} / \kappa, 1)^{\alpha} \times \max (\mathrm{Scr} / \kappa, 1)^{-1.209} \times 0.993^{\text {Age }}[\times 1.018 \text { if female }][\times 1.159 \text { if black }], \text { where } \alpha \text { is } \\
-0.329 \text { for females and }-0.411 \text { for males }\end{array}$ \\
\hline $\begin{array}{l}\text { The CKD-EPI cystatin C } \\
\text { equation }\end{array}$ & $133 \times \min (\text { Scys } / 0.8,1)^{-0.499} \times \max (\text { Scys } / 0.8,1)^{-1.328} \times 0.996^{\text {Age }}[\times 0.932$ if female $]$ \\
\hline $\begin{array}{l}\text { The CKD-EPI } \\
\text { creatinine-cystatin C equation }\end{array}$ & $\begin{array}{l}135 \times \min (\operatorname{Scr} / \kappa, 1)^{\alpha} \times \max (\mathrm{Scr} / \kappa, 1)^{-0.601} \times \min (\mathrm{Scys} / 0.8,1)^{-0.375} \times \max (\mathrm{Scys} / 0.8, \\
1)^{-0.711} \times 0.995^{\text {Age }}[\times 0.969 \text { if female }][\times 1.08 \text { if black }] \text {, where } \alpha \text { is }-0.248 \text { for females and }-0.207 \text { for } \\
\text { males }\end{array}$ \\
\hline
\end{tabular}

$\kappa$ is 0.7 for females and 0.9 for males, max is the maximum of $\mathrm{Scr} / \kappa$ or $1, \mathrm{~min}$ is the minimum of $\mathrm{Scr} / \mathcal{\kappa}$ or 1 , Scr is serum creatinine in $(\mathrm{mg} / \mathrm{dL})$, and $\mathrm{Scys}$ is serum cystatin $\mathrm{C}$ in $(\mathrm{mg} / \mathrm{L})$.

these patients. In our study, we aimed at evaluating the role of serum CysC as a marker of the renal function in patients with liver cirrhosis and, more important, as an indicator of renal impairment at its early stages in such patients. We compared different markers and estimating equations, including serum CysC and CysC-based equations, to the isotopic GFR being used as the gold standard of the actual renal performance.

\section{Methodology}

2.1. Patients. This prospective observational study was conducted on seventy consecutive patients with liver cirrhosis who presented to the Liver Transplantation Unit, National Liver Institute, Menoufia University, as potential candidates for liver transplantation. The diagnosis of cirrhosis was based on a combination of clinical, laboratory, and ultrasonographic findings [20]. Patients aged 18-80 years were eligible for the study if they had serum creatinine less than $1.5 \mathrm{mg} / \mathrm{dL}$. Patients were excluded if they had dehydration, sepsis, or gastrointestinal bleeding during the month before enrollment. The local ethics committee approved the study. A written informed consent was obtained from all participating patients prior to inclusion in the study.

2.2. Baseline Assessment. Included patients were subjected to history taking, clinical examination, abdominal ultrasonography, and baseline laboratory investigations including liver and renal tests, urine analysis, and complete blood count. Child-Pugh [21, 22] and MELD [23] scores were calculated for all patients.

2.3. Assessment of the Renal Function. Enrolled patients

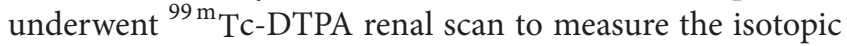
GFR. Serum and urine samples were collected on the same day of the renal scan. Serum samples were used to measure serum creatinine, CysC, and blood urea nitrogen (BUN). The Quantikine Human Cystatin C Immunoassay (R\&D Systems Inc., Minneapolis, MN, USA), an enzyme-linked immunosorbent assay (ELISA), was used to measure serum
CysC on a CX7 analyzer (Beckman, Brea, CA, USA). Creatinine clearance $(\mathrm{CCr})$ was calculated through the following equation: $\mathrm{CCr}=$ (urinary creatinine $\times 24$ hours of urine volume $) /($ serum creatinine $\times 1440)$ [24]. GFR was furthermore calculated using six estimating formulae, CockcroftGault formula [25], the modification of diet in renal disease (MDRD) equations using 4 and 6 variables [26, 27], and Chronic Kidney Disease Epidemiology Collaboration (CKDEPI) equations using creatinine alone (CKD-EPI Cr) [28], CysC alone (CKD-EPI CysC), or both CKD-EPI (Cr-CysC) [29] (Table 1). Isotopic GFR was used as the reference to which all other measures and estimating formulae of the renal function were compared. In this study, the upper limit of normal for serum creatinine was $1.2 \mathrm{mg} / \mathrm{dL}$ and early renal impairment was defined as a GFR of $60-89 \mathrm{~mL} / \mathrm{min}$.

2.4. Statistical Analysis. Data were analyzed using IBM SPSS Statistics for Macintosh, Version 22.0 (IBM Corp., Armonk, NY, USA). Two-tailed $p$ values were considered statistically significant if they were less than 0.05 .

Bivariate correlations were tested using Pearson's $(r)$ and Spearman's (rho) correlation coefficients for parametric and nonparametric measure of statistical dependence, respectively. The coefficient of determination $\left(R^{2}\right)$ was also calculated to measure the percent of variation in one variable which could be predicted by the variation in the other variable. $R^{2}$ closer to 1 indicates a better model fit.

The ability of the studied tests and formulae to discriminate early renal impairment was evaluated using the area under the receiver operating characteristic (ROC) curve (AUC). A cutoff was then proposed to each test or formula with the most acceptable sensitivity and specificity. The following tests of diagnostic accuracy were calculated for the measures and estimates of the renal function with a $95 \%$ confidence interval: sensitivity, specificity, positive predictive value (PPV), and negative predictive value (NPV).

Bland-Altman plot was used to analyze the agreement between isotopic GFR and the other mathematical formulae of GFR. One sample $t$-test was used to test for significance of the difference between each of these formulae and isotopic GFR. 
TABLE 2: Baseline characteristics of the enrolled patients.

\begin{tabular}{lcc}
\hline & Mean \pm SD & Range \\
\hline Age (years) & $47.4 \pm 9.3$ & $18-75$ \\
Weight (kg) & $78.2 \pm 14.7$ & $48-110$ \\
Total bilirubin (mg/dL) & $3.9 \pm 3.2$ & $0.6-17.5$ \\
Albumin (g/dL) & $2.6 \pm 0.5$ & $1.7-3.5$ \\
Isotopic GFR (mL/min) & $84.5 \pm 16.6$ & $53-133$ \\
BUN (mg/dL) & $19.3 \pm 10.8$ & $4.7-72.3$ \\
Creatinine (mg/dL) & $0.8 \pm 0.3$ & $0.3-1.4$ \\
Creatinine clearance (mL/min) & $82.4 \pm 31.3$ & $24-199$ \\
Cystatin C (mg/L) & $1.9 \pm 1$ & $0.3-4.5$ \\
Child-Pugh score & $10.7 \pm 1.7$ & $7-14$ \\
MELD score & $16.2 \pm 4.9$ & $8-31$ \\
MDRD 4 (mL/min) & $119.2 \pm 63.5$ & $45.7-367$ \\
MDRD 6 (mL/min) & $97.4 \pm 50.4$ & $28.4-291.7$ \\
Cockcroft-Gault formula (mL/min) & $132.9 \pm 65$ & $47.9-421.1$ \\
CKD-EPI-Cr (mL/min) & $101.0 \pm 26.7$ & $47.1-175.6$ \\
CKD-EPI-CysC (mL/min) & $52 \pm 40$ & $11.4-185.9$ \\
CKD-EPI-Cr-CysC (mL/min) & $61.5 \pm 26.3$ & $29.7-154.8$ \\
\hline
\end{tabular}

BUN, blood urea nitrogen; CKD-EPI, Chronic Kidney Disease Epidemiology Collaboration; GFR, glomerular filtration rate; MDRD, modification of diet in renal disease equation with 4 and 6 variables; MELD, model for endstage liver disease; SD, standard deviation.

\section{Results}

The baseline characteristics and demographic and clinical data of the enrolled patients are presented in Tables 2 and 3. The study sample had predominance of males (87.1\%). Etiology of the liver disease was most commonly chronic hepatitis C infection (72.9\%). About $29 \%$ of the patients had hepatocellular carcinoma (HCC). Most patients were ChildPugh class C (74.3\%) and had moderate ascites (41.4\%). About $64 \%$ of the patients had early renal impairment. None of the patients had proteinuria or other urine abnormalities.

Different measures and estimates of the renal function were correlated with the isotopic GFR using Pearson's correlation (Table 4). Serum CysC and CKD-EPI equations using $\mathrm{CysC}$ alone or both creatinine and $\mathrm{CysC}$ had the highest and most significant correlation coefficient $(r=0.437,0.473$, and 0.474 , resp., $p<0.0001$ ) (Figure 1). CKD-EPI (Cr-CysC) had the highest $R^{2}$ among all other measures.

The discriminating ability of the studied measures and estimates of the renal function in detecting early renal impairment was assessed by plotting the ROC curves (Figure 2). Table 5 presents the AUC for each of the measures and formulae. CKD-EPI (Cr-CysC) had the largest AUC $(0.808, p<$ 0.0001 ). We then used the ROC curves' coordinates to define cutoff values with acceptable sensitivity and specificity for the studied tests and formulae. Table 6 shows the sensitivity, specificity, PPV, and NPV for the different measures and formulae in detecting early renal impairment according to these cutoff values. At a cutoff value of $1.2 \mathrm{mg} / \mathrm{L}, \mathrm{CysC}$ was $89.6 \%$ sensitive and $63.6 \%$ specific in detecting early renal impairment with PPV of $84.3 \%$ and NPV of $73.7 \%$.
TABLE 3: Demographic and clinical data of the enrolled patients.

\begin{tabular}{lc}
\hline & $n(\%)$ \\
\hline Sex & \\
Males & $61(87.1)$ \\
Females & $9(12.9)$ \\
Etiology of liver disease & \\
Chronic hepatitis C & $51(72.9)$ \\
Chronic hepatitis B & $12(17.1)$ \\
Budd-Chiari syndrome & $2(2.9)$ \\
Unknown etiology & $5(7.1)$ \\
HCC & \\
No & $50(71.4)$ \\
Yes & $20(28.6)$ \\
Ascites & \\
No & $9(12.9)$ \\
Mild & $23(32.8)$ \\
Moderate & $29(41.4)$ \\
Marked & $9(12.9)$ \\
Child-Pugh class & \\
B & $18(25.7)$ \\
C & $52(74.3)$ \\
Renal impairment & \\
No (GFR $\geq 90 \mathrm{~mL} / \mathrm{min})$ & $22(31.4)$ \\
Early (GFR 60-89 mL/min) & $45(64.3)$ \\
Advanced (GFR 30-59 mL/min) & $3(4.3)$ \\
\hline GFR, glomerular filtration rate; HCC, hepatocellular carcinoma. &
\end{tabular}

TABLE 4: Pearson's correlation of the variables with the isotopic GFR.

\begin{tabular}{lcc}
\hline & $r$ & $p$ \\
\hline 1/creatinine $\left(\mathrm{mg} / \mathrm{dL}^{-1}\right)$ & 0.287 & 0.016 \\
Creatinine clearance $(\mathrm{mL} / \mathrm{min})$ & 0.367 & 0.002 \\
1/cystatin C $\left(\mathrm{mg} / \mathrm{L}^{-1}\right)$ & 0.437 & $<0.0001$ \\
MDRD 4 & 0.260 & 0.030 \\
MDRD 6 & 0.286 & 0.017 \\
Cockcroft-Gault formula & 0.198 & 0.100 \\
CKD-EPI-Cr & 0.247 & 0.039 \\
CKD-EPI-CysC & 0.473 & $<0.0001$ \\
CKD-EPI-Cr-CysC & 0.474 & $<0.0001$ \\
\hline
\end{tabular}

CKD-EPI, Chronic Kidney Disease Epidemiology Collaboration; Cr, creatinine; $\mathrm{Cys} C$, cystatin $\mathrm{C}$; MDRD, modification of diet in renal disease equation with 4 and 6 variables; $r$, Pearson's correlation coefficient.

The Bland-Altman plot was used to describe agreement between isotopic GFR and different mathematical formulae (Table 7 and Figure 3). The mean difference with CCr was 2.0429 , which was not statistically significant $(p=0.565)$.

\section{Discussion}

This study showed that serum CysC and $\mathrm{CysC}$-based equations were the best measures that correlated with the isotopic GFR, compared to other measures and equations that have been evaluated in the study. This confirms the results of the 

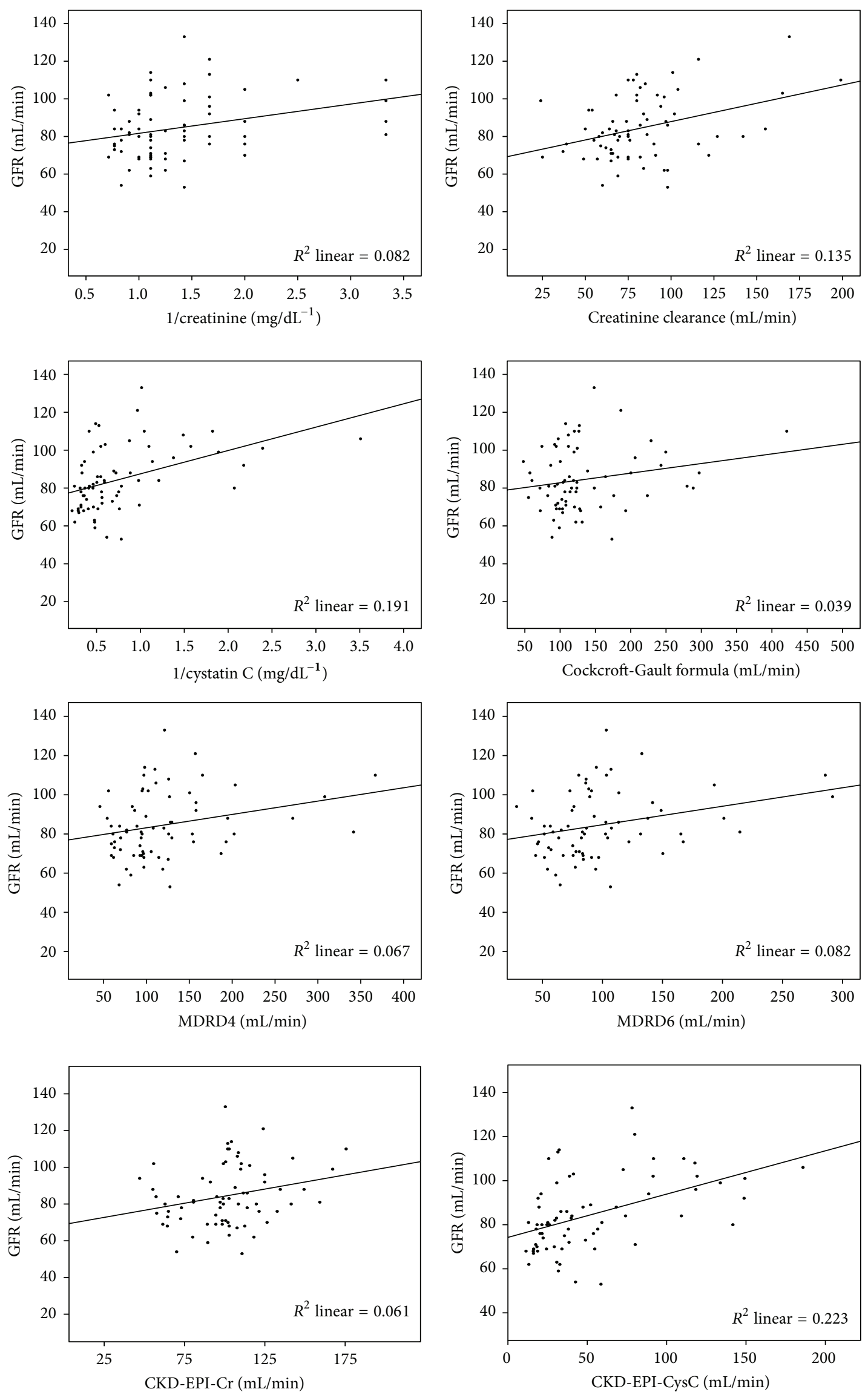

Figure 1: Continued. 


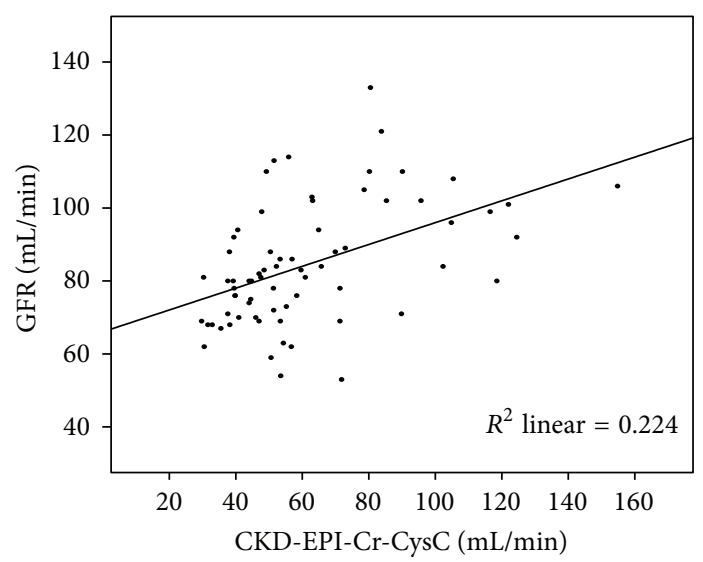

FIGURE 1: Scatter plots of the isotopic glomerular filtration rate (GFR) versus 1/creatinine, creatinine clearance, 1/cystatin C, Cockcroft-Gault formula, MDRD equations with 4 and 6 variables, CKD-EPI-Cr, CKD-EPI-CysC, and CKD-EPI-Cr-CysC.

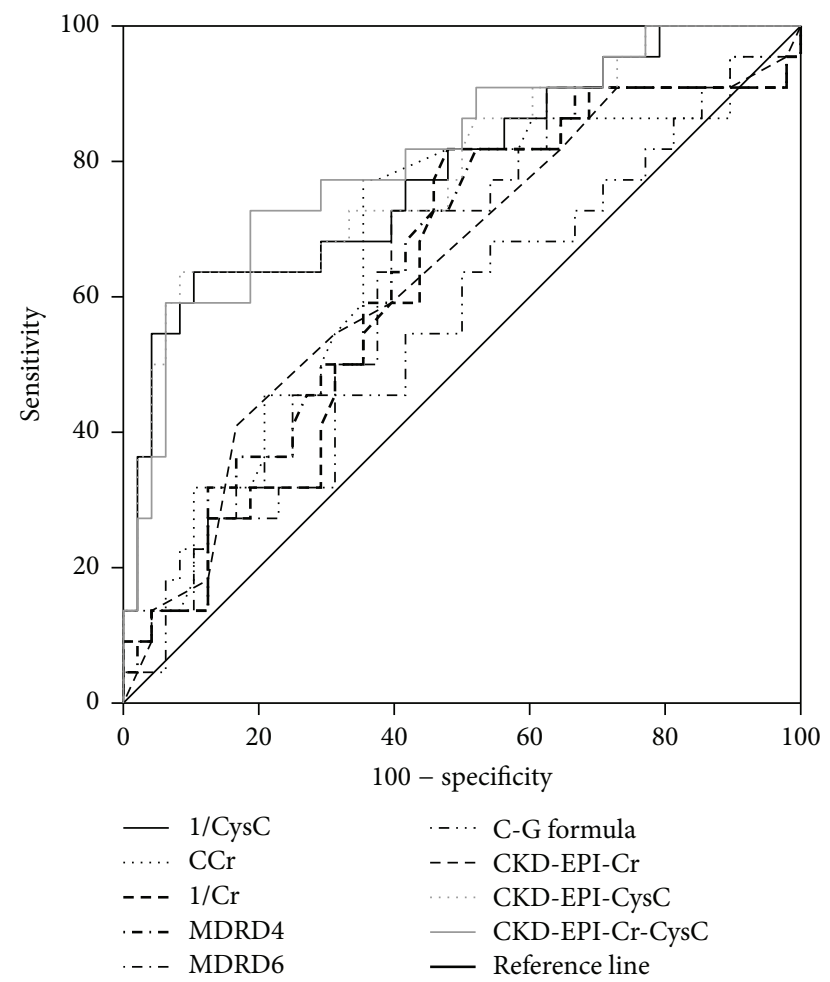

FIgURE 2: ROC curves of $1 /$ creatinine, creatinine clearance, 1 /cystatin C, MDRD equations with 4 and 6 variables, Cockcroft-Gault formula, CKD-EPI-Cr, CKD-EPI-CysC, and CKD-EPI-Cr-CysC for detecting early renal impairment.

previous studies that came up with similar conclusions $[18$, 30-32]. Several studies have reported the superior diagnostic accuracy of serum CysC and CysC-based formulae over other markers and equations in detecting moderate and severe renal impairment in patients with liver cirrhosis [18, 33, 34]. However, since patients with liver cirrhosis are extremely sensitive to the modest decreases in the GFR that can markedly impact their survival [35], it is of great clinical importance to identify markers that can detect renal impairment at its
TABLE 5: Area under the curve for detecting early renal impairment for studied tests and formulae.

\begin{tabular}{lccc}
\hline Variable & AUC & $95 \%$ CI & $p$ \\
\hline 1/Cr & 0.642 & $0.500-0.784$ & 0.058 \\
CCr & 0.674 & $0.533-0.815$ & 0.020 \\
1/CysC & 0.785 & $0.663-0.907$ & $<0.0001$ \\
MDRD 4 & 0.646 & $0.506-0.787$ & 0.051 \\
MDRD 6 & 0.644 & $0.503-0.784$ & 0.054 \\
C-G formula & 0.562 & $0.413-0.710$ & 0.411 \\
CKD-EPI-Cr & 0.632 & $0.491-0.773$ & 0.078 \\
CKD-EPI-CysC & 0.788 & $0.667-0.909$ & $<0.0001$ \\
CKD-EPI-Cr-CysC & 0.808 & $0.695-0.921$ & $<0.0001$ \\
\hline
\end{tabular}

AUC, area under the curve; C-G formula, Cockcroft-Gault formula; CKDEPI, Chronic Kidney Disease Epidemiology Collaboration; CI, confidence interval; $\mathrm{Cr}$, creatinine; $\mathrm{CCr}$, creatinine clearance; CysC, cystatin C; MDRD, modification of diet in renal disease equation with 4 and 6 variables.

early stage in these patients. Using Pearson's correlation, our study found that CKD-EPI (Cr-CysC) formula was the most accurate marker, with the largest AUC, in detecting early renal impairment in patients with liver cirrhosis. This finding is novel, putting CysC and CysC-based equations on the top of the list of markers and estimating formulae of the renal function in such patients. The results also showed that serum CysC was the most sensitive $(89.6 \%)$ measure in detecting early renal impairment at a cutoff value of $1.2 \mathrm{mg} / \mathrm{L}$ with acceptable specificity (63.6\%).

One strong limitation of serum creatinine and creatininebased equations is that serum creatinine lags behind a decreasing GFR [36]. So they are not accurately reflecting the present status of the renal function of the patient, a limitation that has been overcome by serum $\mathrm{Cys} C$ that proved to accurately reflect the early stages of renal impairment according to the results of this study.

However, Bland-Altman plot analysis showed disputing results. Estimated CCr was the only measurement that had significant agreement with isotopic GFR. This contradiction may be explained as a correlation coefficient between two 

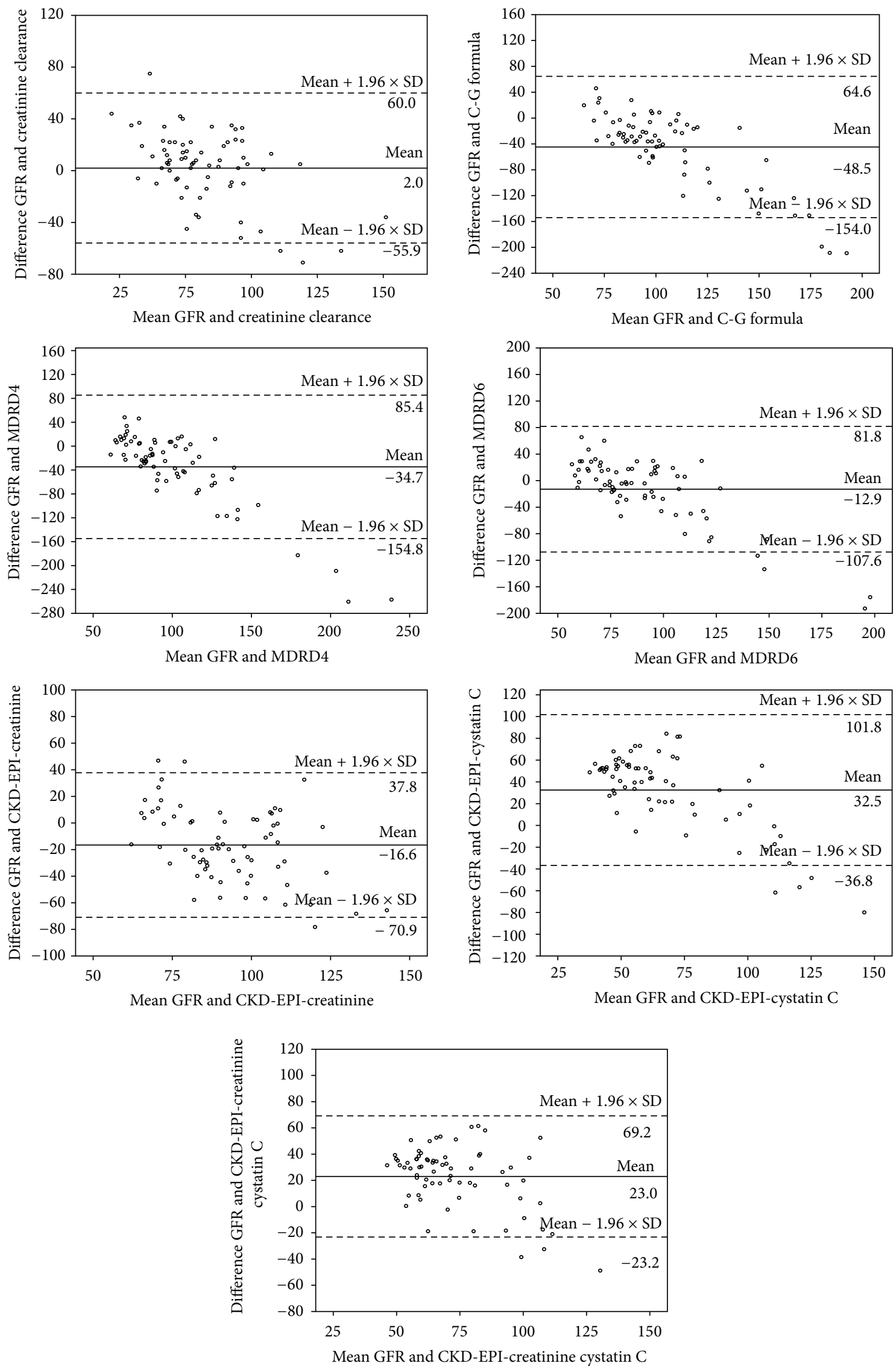

FIGURE 3: Bland-Altman plot for the agreement between isotopic GFR and the other GFR estimating formulae. 
TABLE 6: Cutoffs of measures and estimates for detection of early renal impairment with their measures of diagnostic accuracy.

\begin{tabular}{|c|c|c|c|c|c|}
\hline & Cutoff of early RI & Sensitivity (\%) & Specificity (\%) & PPV (\%) & NPV (\%) \\
\hline $\mathrm{Cr}$ & $0.75 \mathrm{mg} / \mathrm{dL}$ & 68.8 & 54.5 & 76.7 & 44.4 \\
\hline $\mathrm{CCr}$ & $77 \mathrm{~mL} / \mathrm{min}$ & 77.3 & 64.6 & 54.5 & 60.9 \\
\hline CysC & $1.2 \mathrm{mg} / \mathrm{L}$ & 89.6 & 63.6 & 84.3 & 73.7 \\
\hline MDRD 4 & $96.8 \mathrm{~mL} / \mathrm{min}$ & 72.7 & 54.2 & 57.3 & 62.7 \\
\hline MDRD 6 & $85.5 \mathrm{~mL} / \mathrm{min}$ & 72.7 & 60.4 & 54.6 & 59.2 \\
\hline C-G formula & $119.3 \mathrm{~mL} / \mathrm{min}$ & 54.5 & 58.3 & 48.3 & 47.8 \\
\hline CKD-EPI-Cr & $99.8 \mathrm{~mL} / \mathrm{min}$ & 77.3 & 55.2 & 63.3 & 70.9 \\
\hline CKD-EPI-CysC & $38.7 \mathrm{~mL} / \mathrm{min}$ & 72.7 & 66.7 & 68.6 & 71.0 \\
\hline CKD-EPI-Cr-CysC & $52.8 \mathrm{~mL} / \mathrm{min}$ & 77.3 & 61.4 & 66.7 & 73.0 \\
\hline
\end{tabular}

C-G formula, Cockcroft-Gault formula; CKD-EPI, Chronic Kidney Disease Epidemiology Collaboration; Cr, creatinine; CCr, creatinine clearance; CysC, cystatin C; MDRD, modification of diet in renal disease equation with 4 and 6 variables; NPV, negative predictive value; PPV, positive predictive value; RI, renal impairment.

TABLE 7: Bland-Altman plot analysis for the agreement between isotopic GFR and the other GFR estimating formulae.

\begin{tabular}{lccc}
\hline & Mean \pm SD & $p$ & $95 \%$ CI \\
\hline CCr & $2.04 \pm 29.6$ & 0.565 & $-5.0-9.1$ \\
C-G formula & $-44.7 \pm 55.8$ & $<0.0001$ & $-58.1--31.3$ \\
MDRD 4 & $-34.7 \pm 61.3$ & $<0.0001$ & $-49.3--20.1$ \\
MDRD 6 & $-12.9 \pm 48.3$ & 0.028 & $-24.5--1.4$ \\
CKD-EPI-Cr & $-16.6 \pm 27.7$ & $<0.0001$ & $-23.2--9.9$ \\
CKD-EPI-CysC & $32.5 \pm 35.3$ & $<0.0001$ & $24.1-40.9$ \\
CKD-EPI-Cr-CysC & $23 \pm 23.6$ & $<0.0001$ & $17.4-28.6$ \\
\hline
\end{tabular}

C-G formula, Cockcroft-Gault formula; CKD-EPI, Chronic Kidney Disease Epidemiology Collaboration; $\mathrm{Cr}$, creatinine; $\mathrm{CCr}$, creatinine clearance; $\mathrm{CysC}$, cystatin C; MDRD, modification of diet in renal disease equation with 4 and 6 variables.

measures might be highly correlated; yet, there could be substantial differences in the two measurements across their range of values. Also, results of Bland-Altman method might be disturbed when there is heterogeneous bias with significant heteroscedastic error [37].

The results of this study are robust even though the sample size was relatively small because we have used the isotopic GFR as the reference to which all other measures and formulae were compared. Therefore, in contrast to some previous studies that used $\mathrm{CCr}$ as reference [33, 38], the results of our study can be generalized over a larger scale. Still, we recommend further studies to be designed to confirm and validate the results of this study. Future studies should formulate a general predictive model of the renal function in patients with liver cirrhosis based on serum CysC levels, a model that can make the isotopic GFR dispensable.

In conclusion, finding a good marker of the renal function is crucial to the survival of patients with liver cirrhosis, given the huge impact of the renal impairment on the prognosis of these patients. Our study found that serum CysC and CysC-based formulae were not only the best measures that reflected the actual renal performance in cirrhotic patients, but also the most accurate ones in detecting early stages of renal impairment in these patients.

\section{Conflict of Interests}

The authors declare that there is no conflict of interests regarding the publication of this paper.

\section{References}

[1] A. Slack, A. Yeoman, and J. Wendon, "Renal dysfunction in chronic liver disease," Critical Care, vol. 14, no. 2, article 214, 2010.

[2] V. Arroyo, J. Fernandez, and P. Ginès, "Pathogenesis and treatment of hepatorenal syndrome," Seminars in Liver Disease, vol. 28, no. 1, pp. 81-95, 2008.

[3] T. Takabatake, H. Ohta, Y.-I. Ishida, H. Hara, Y. Ushiogi, and N. Hattori, "Low serum creatinine levels in severe hepatic disease," Archives of Internal Medicine, vol. 148, no. 6, pp. 1313-1315, 1988.

[4] A. J. Slack and J. Wendon, "The liver and kidney in critically ill patients," Blood Purification, vol. 28, no. 2, pp. 124-134, 2009.

[5] E. Cholongitas, V. Shusang, L. Marelli et al., "Review article: renal function assessment in cirrhosis-difficulties and alternative measurements," Alimentary Pharmacology and Therapeutics, vol. 26, no. 7, pp. 969-978, 2007.

[6] N. L. Proulx, A. Akbari, A. X. Garg, A. Rostom, J. Jaffey, and H. D. Clark, "Measured creatinine clearance from timed urine collections substantially overestimates glomerular filtration rate in patients with liver cirrhosis: a systematic review and individual patient meta-analysis," Nephrology Dialysis Transplantation, vol. 20, no. 8, pp. 1617-1622, 2005.

[7] J. MacAulay, K. Thompson, B. A. Kiberd, D. C. Barnes, and K. M. Peltekian, "Serum creatinine in patients with advanced liver disease is of limited value for identification of moderate renal dysfunction: are the equations for estimating renal function better?" Canadian Journal of Gastroenterology, vol. 20, no. 8, pp. 521-526, 2006.

[8] L. A. Stevens, J. Coresh, T. Greene, and A. S. Levey, "Assessing kidney function-measured and estimated glomerular filtration rate," The New England Journal of Medicine, vol. 354, no. 23, pp. 2473-2483, 2006.

[9] Y. Kandasamy, R. Smith, and I. M. R. Wright, "Measuring cystatin $\mathrm{C}$ to determine renal function in neonates," Pediatric Critical Care Medicine, vol. 14, no. 3, pp. 318-322, 2013.

[10] R. B. Dhia, I. Hellara, O. Harzallah et al., "Evaluation of the renal function in type 2 diabetes: clearance calculation or cystatin C?" Annales de Biologie Clinique, vol. 70, no. 3, pp. 287-294, 2012. 
[11] H.-X. Guo, C.-H. Wang, Z.-Q. Li et al., "The application of serum cystatin $\mathrm{C}$ in estimating the renal function in women with preeclampsia," Reproductive Sciences, vol. 19, no. 7, pp. 712717, 2012.

[12] S. Niemczyk, M. Piotrowska, and K. Szamotulska, "Usefulness of creatinine and cystatin $\mathrm{C}$ in the assessment of renal function in chronic kidney disease and coexisting diseases," Polski Merkuriusz Lekarski, vol. 32, no. 191, pp. 313-317, 2012.

[13] M. M. Sagheb, S. Namazi, B. Geramizadeh, A. Karimzadeh, M. B. Oghazian, and I. Karimzadeh, "Serum cystatin C as a marker of renal function in critically Ill patients with normal serum creatinine," Nephro-Urology Monthly, vol. 6, no. 2, Article ID e15224, 2014.

[14] Y. Suzuki, K. Matsushita, T. Yoshida, Y. Sawabe, and F. Nomura, "Clinical validity of renal function markers including serum cystatin C on chronic kidney disease classification," Rinsho Byori, vol. 59, no. 4, pp. 345-351, 2011.

[15] Y. Suzuki, M. Seimiya, T. Yoshida, Y. Sawabe, K. Matsushita, and F. Nomura, "Usefulness of the standardized serum cystatin C reagent in evaluation of renal function," Rinsho Byori, vol. 61, no. 8, pp. 665-670, 2013.

[16] K.-S. Woo, J.-L. Choi, B.-R. Kim, J.-E. Kim, and J.-Y. Han, "Clinical usefulness of serum cystatin $\mathrm{C}$ as a marker of renal function," Diabetes and Metabolism Journal, vol. 38, no. 4, pp. 278-284, 2014.

[17] Đ. Ćulafić, "Role of cystatin $\mathrm{C}$ and renal resistive index in assessment of renal function in patients with liver cirrhosis," World Journal of Gastroenterology, vol. 20, no. 21, pp. 6573-6579, 2014.

[18] D. J. Kim, H. S. Kang, H. S. Choi et al., "Serum cystatin C level is a useful marker for the evaluation of renal function in patients with cirrhotic ascites and normal serum creatinine levels," The Korean Journal of Hepatology, vol. 17, no. 2, pp. 130-138, 2011.

[19] Y. Ustundag, K. Hekimoğlu, S. Ilikhan, G. Zaimoğlu, S. Acikgöz, and S. Aydemir, "Serum glucagon and cystatin C levels with renal doppler sonography findings in non-azotemic liver cirrhosis cases," Hepato-Gastroenterology, vol. 58, no. 107-108, pp. 926-931, 2011.

[20] D. Schuppan and N. H. Afdhal, "Liver cirrhosis," The Lancet, vol. 371, no. 9615, pp. 838-851, 2008.

[21] C. G. Child and J. G. Turcotte, "Surgery and portal hypertension," Major problems in clinical surgery, vol. 1, pp. 1-85, 1964.

[22] R. N. H. Pugh, I. M. Murray Lyon, and J. L. Dawson, "Transection of the oesophagus for bleeding oesophageal varices," British Journal of Surgery, vol. 60, no. 8, pp. 646-649, 1973.

[23] R. Wiesner, E. Edwards, R. Freeman et al., "Model for endstage liver disease (MELD) and allocation of donor livers," Gastroenterology, vol. 124, no. 1, pp. 91-96, 2003.

[24] B. A. C. van Acker, G. C. M. Koomen, M. G. Koopman, D. R. de Waart, and L. Arisz, "Creatinine clearance during cimetidine administration for measurement of glomerular filtration rate," The Lancet, vol. 340, no. 8831, pp. 1326-1329, 1992.

[25] D. W. Cockcroft and M. H. Gault, "Prediction of creatinine clearance from serum creatinine," Nephron, vol. 16, no. 1, pp. 3141, 1976.

[26] A. S. Levey, J. P. Bosch, J. B. Lewis, T. Greene, N. Rogers, and D. Roth, "A more accurate method to estimate glomerular filtration rate from serum creatinine: a new prediction equation," Annals of Internal Medicine, vol. 130, no. 6, pp. 461-470, 1999.

[27] National Kidney Foundation, "K/DOQI clinical practice guidelines for chronic kidney disease: evaluation, classification, and stratification," American Journal of Kidney Diseases, vol. 39, no. 2, supplement 1, pp. S1-S266, 2002.

[28] A. S. Levey, L. A. Stevens, C. H. Schmid et al., "A new equation to estimate glomerular filtration rate," Annals of Internal Medicine, vol. 150, no. 9, pp. 604-612, 2009.

[29] L. A. Inker, C. H. Schmid, H. Tighiouart et al., "Estimating glomerular filtration rate from serum creatinine and cystatin C," The New England Journal of Medicine, vol. 367, no. 1, pp. 20-29, 2012.

[30] H. S. Ahn, Y. S. Kim, S. G. Kim et al., "Cystatin C is a good predictor of hepatorenal syndrome and survival in patients with cirrhosis who have normal serum creatinine levels," HepatoGastroenterology, vol. 59, no. 116, pp. 1168-1173, 2012.

[31] Y. Ustundag, U. Samsar, S. Acikgoz et al., "Analysis of glomerular filtration rate, serum cystatin C levels, and renal resistive index values in cirrhosis patients," Clinical Chemistry and Laboratory Medicine, vol. 45, no. 7, pp. 890-894, 2007.

[32] S. Demirtaş, A. Bozbaş, A. Akbay, Y. Yavuz, and L. Karaca, "Diagnostic value of serum cystatin C for evaluation of hepatorenal syndrome," Clinica Chimica Acta, vol. 311, no. 2, pp. 81$89,2001$.

[33] E. Randers, P. Ivarsen, E. J. Erlandsen et al., "Plasma cystatin C as a marker of renal function in patients with liver cirrhosis," Scandinavian Journal of Clinical and Laboratory Investigation, vol. 62, no. 2, pp. 129-134, 2002.

[34] M. Adachi, A. Tanaka, M. Aiso, Y. Takamori, and H. Takikawa, "Benefit of cystatin C in evaluation of renal function and prediction of survival in patients with cirrhosis," Hepatology Research, 2015.

[35] P. Ginès and R. W. Schrier, "Renal failure in cirrhosis," The New England Journal of Medicine, vol. 361, no. 13, pp. 1279-1290, 2009.

[36] S. Herget-Rosenthal, J. Metzger, A. Albalat, V. Bitsika, and H. Mischak, "Proteomic biomarkers for the early detection of acute kidney injury," Prilozi, vol. 33, no. 1, pp. 27-48, 2012.

[37] D. P. O'Connor, M. T. Mahar, M. S. Laughlin, and A. S. Jackson, "The Bland-Altman method should not be used in regression cross-validation studies," Research Quarterly for Exercise and Sport, vol. 82, no. 4, pp. 610-616, 2011.

[38] A. L. Gerbes, V. Gülberg, M. Bilzer, and M. Vogeser, "Evaluation of serum cystatin $\mathrm{C}$ concentration as a marker of renal function in patients with cirrhosis of the liver," Gut, vol. 50, no. 1, pp. 106$110,2002$. 


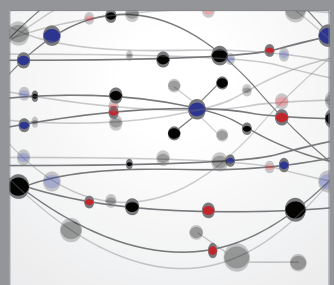

The Scientific World Journal
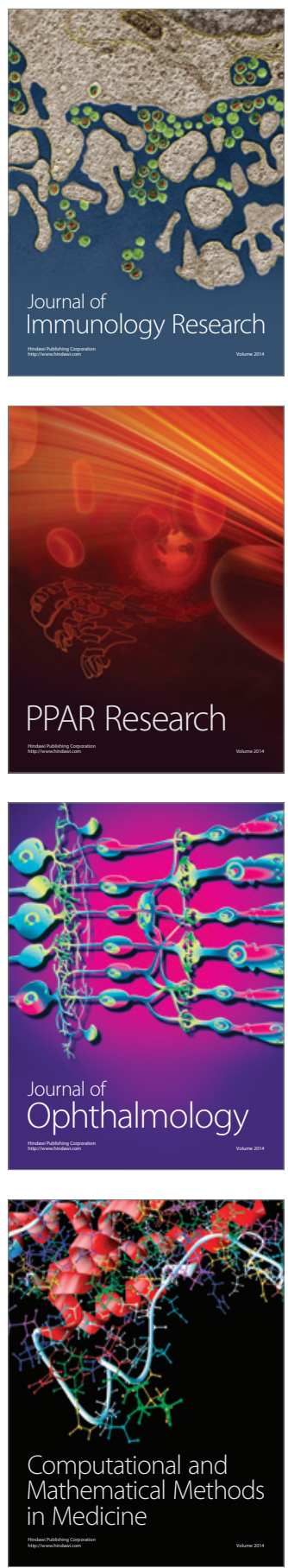

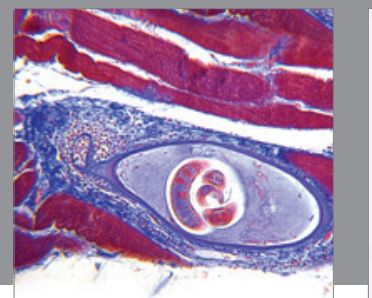

Gastroenterology

Research and Practice
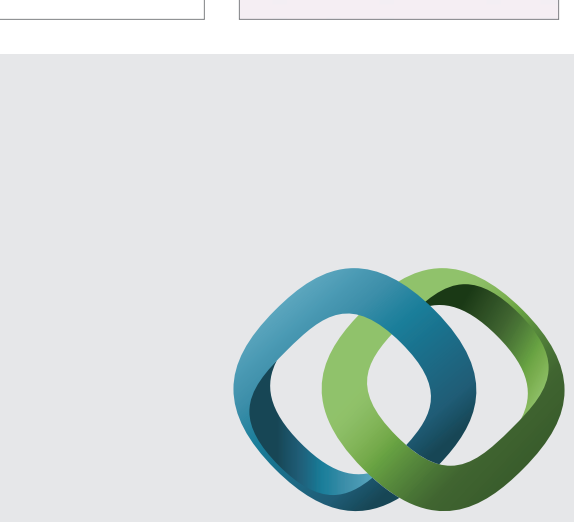

\section{Hindawi}

Submit your manuscripts at

http://www.hindawi.com
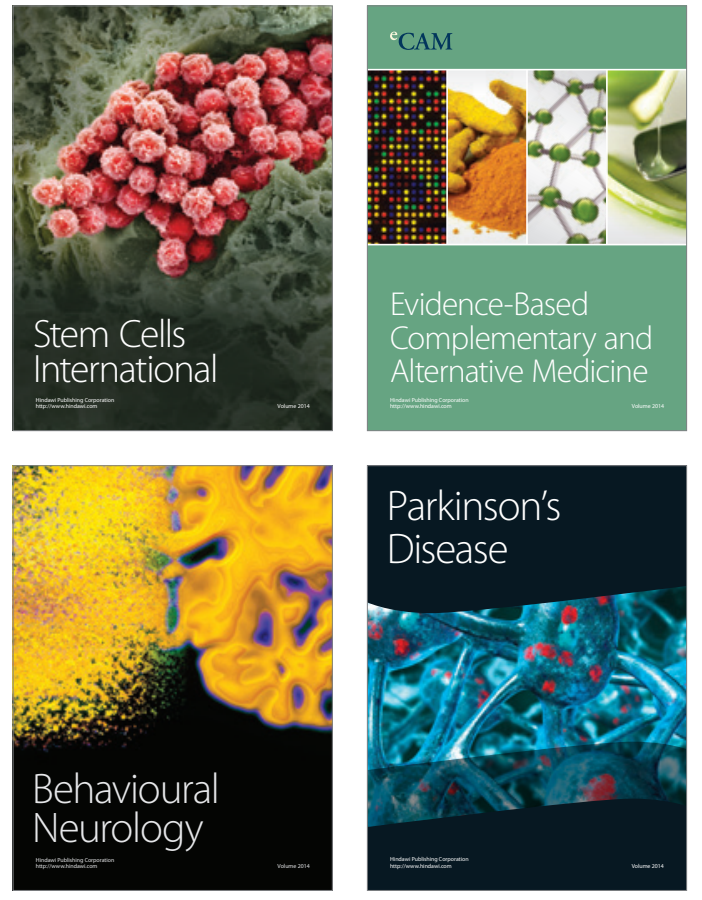
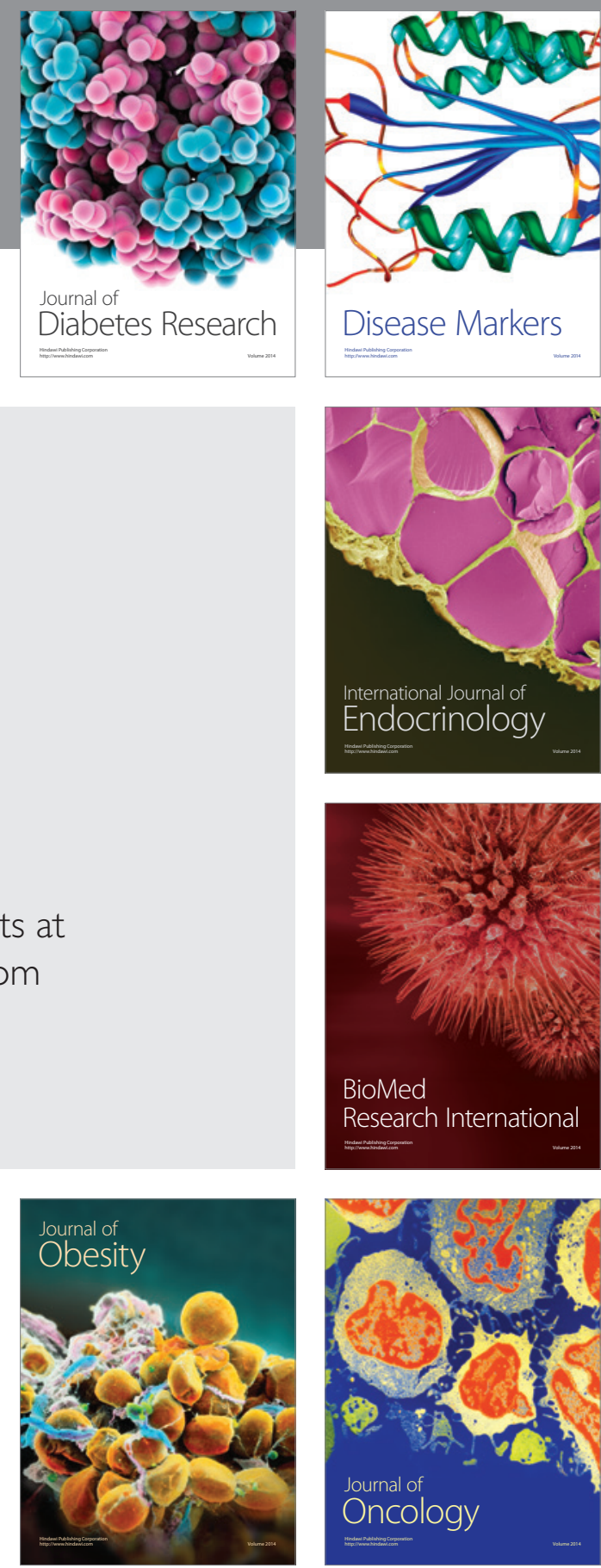

Disease Markers
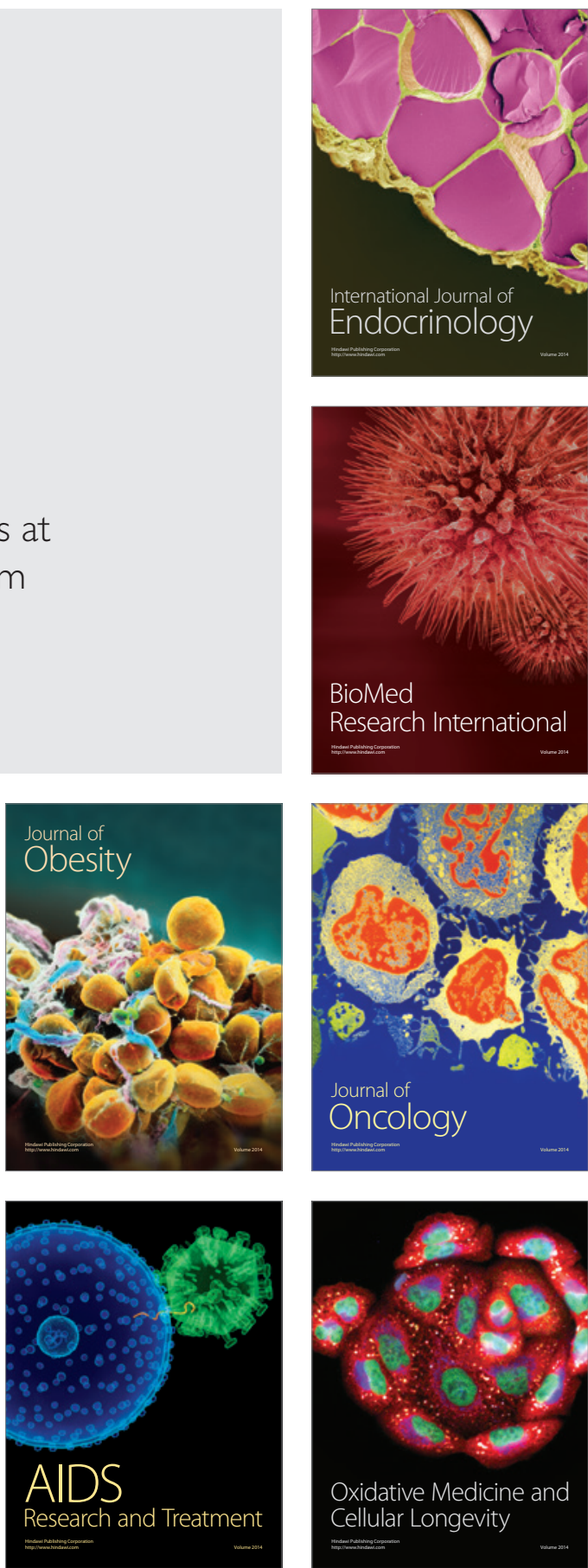